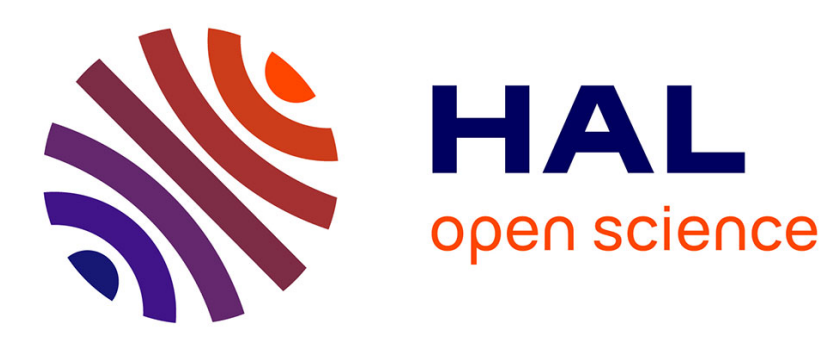

\title{
Numerical stud of glare spot phase Doppler anemometry
}

Camille Hespel, Kuan Fang Ren, Gérard Gréhan, Fabrice R. A. Onofri

\section{To cite this version:}

Camille Hespel, Kuan Fang Ren, Gérard Gréhan, Fabrice R. A. Onofri. Numerical stud of glare spot phase Doppler anemometry. Optics Communications, 2008, 281, pp.1375-1383.

10.1016/j.optcom.2007.11.034 . hal-00601955

\section{HAL Id: hal-00601955 \\ https://hal.science/hal-00601955}

Submitted on 21 Jun 2011

HAL is a multi-disciplinary open access archive for the deposit and dissemination of scientific research documents, whether they are published or not. The documents may come from teaching and research institutions in France or abroad, or from public or private research centers.
L'archive ouverte pluridisciplinaire HAL, est destinée au dépôt et à la diffusion de documents scientifiques de niveau recherche, publiés ou non, émanant des établissements d'enseignement et de recherche français ou étrangers, des laboratoires publics ou privés. 
Elsevier Editorial System(tm) for Optics Communications

Manuscript Draft

Manuscript Number: SK-4340R2

Title: Numerical study of Glare Spot Phase Doppler Anemometry

Article Type: Journal Article

Section/Category: I. Physical Optics

Keywords:

Corresponding Author: Dr. Kuan Fang Ren,

Corresponding Author's Institution: CNRS-Universite et INSA de Rouen

First Author: Camille Hespel, Ph. D

Order of Authors: Camille Hespel, Ph. D; Kuan Fang Ren; Gerard Grehan, Directeur de Recherche; F. Onofri

Manuscript Region of Origin:

Abstract: The Phase Doppler anemometry (PDA) has been developed to measure simultaneously the velocity and the size of droplets. When the concentration of particles is high, tightly focused beams must be used, as in the Dual Burst PDA. The latter permits an access to the refractive index of the particle, but the effect of wave front curvature of the incident beams becomes evident. In this paper, we introduce a Glare Spot Phase Doppler Anemometry which uses two large beams. The images of the particle formed by the reflected and refracted light, known as glare spots, are separated in space. When a particle passes through the probe volume, the two parts in a signal obtained by a detector in forward direction are then separated in time. If two detectors are used the phase differences and the intensity ratios between two signals, the distance between the reflected and refracted spots can be obtained. These measured values provide information about the particle diameter and its refractive index, as well as its two velocity components. This paper is devoted to the numerical study of such a configuration with two theoretical models: geometrical optics and rigorous electromagnetism solution. 



\title{
Numerical study of Glare Spot Phase Doppler Anemometry
}

\author{
C. Hespel ${ }^{1)}$, K. F. Ren ${ }^{1) *}$, G. Gréhan ${ }^{1)}$ and F. Onofri ${ }^{2)}$ \\ 1) UMR 6614/CORIA, CNRS/Université et INSA de Rouen, \\ BP 12, 76801 Saint Etienne du Rouvray
}

\section{2) IUSTI, UMR 6595 CNRS/Université de Provence, Polytech' Marseille-DME,} Technopôle Château-Gombert, 5 rue E. Fermi, 13453 Marseille

\begin{abstract}
\end{abstract}
The Phase Doppler anemometry has (PDA) been developed to measure simultaneously the velocity and the size of droplets. When the concentration of particles is high, tightly focused beams must be used, as in the Dual Burst PDA. The latter permits an access to the refractive index of the particle, but the effect of wave front curvature of the incident beams becomes evident. In this paper, we introduce a Glare Spot Phase Doppler Anemometry which uses two large beams. The images of the particle formed by the reflected and refracted light, known as glare spots, are separated in space. When a particle passes through the probe volume, the two parts in a signal obtained by a detector in forward direction are then separated in time. If two detectors are used the phase differences and the intensity ratios between two signals, the distance between the reflected and refracted spots can be obtained. These measured values provide information about the particle diameter and its refractive index, as well as its two velocity components. This paper is devoted to the numerical study of such a configuration with two theoretical models: geometrical optics and rigorous electromagnetism solution.

\section{Introduction}

In the research of two-phase flow, the measurement of the velocity and the size of the droplets is essential. The Phase Doppler anemometry (PDA) is one of the most important and the most widely used techniques to reach this end because it gives the correlation between the size and the velocity. But when the concentration of the particles is high, tightly focused beams must

\footnotetext{
${ }^{*}$ Corresponding author. Tel. : +33(0)232953743, Fax : +33(0)232953794. E-mail : fang.ren@coria.fr (K. F. Ren).
} 
be used to ensure that there is only one particle in the probe volume. The wave front effect can

then be considerable. Furthermore, in certain circumstances, as in spray combustion or mixing, we need also to have access to the particle temperature/refractive index or mixing fraction.

With an elastic light scattering based technique such as PDA or rainbow refractometry, the only way to get information about the particle temperature or its material properties is by the measurement of its refractive index. The rainbow refractometry can measure precisely the refractive index (resolution up to $2.010^{-3}$ for nearly mono-dispersed droplets $^{[1]}$ and the mean refractive index for polydispersed clouds ${ }^{[2][3]}$ ). But it does not give information about the velocity of the particles.

There exist also non elastic light scattering based techniques, such as the Laser Induced Fluorescence (LIF) ${ }^{[4][5][6]}$ or the Raman spectroscopy, which allow to measure the temperature and the particle composition. But these diagnostics are quite difficult to implement. LIF, for example, is based on the dependence of fluorescence spectra on the temperature and the dye must be added in the droplets. The properties of fluorescence depends also strongly on the pressure and composition of the droplets. Furthermore, those techniques require complementary methods to access to the particle size and diameter (such as PDA) ${ }^{[7]}$.

Then the in situ estimation of the refractive index of the scattering particles with the PDA technique is a challenge both to increase the PDA size measurement accuracy and to extract correlated information on the particle velocity, size, temperature and/or composition.

For this purpose, different techniques have been developed ${ }^{[7]}$ : extended $\operatorname{PDA}^{[8][9]}$, Dual Mode PDA ${ }^{[10]}$, Dual Burst PDA ${ }^{[1][12]}$, coupling of PDA and rainbow refractometry ${ }^{[13]}$, coupling of PDA and $\mathrm{LIF}^{[14]}$ which give velocity, size and refractive index of droplets. Among them, Dual Burst technique is based on the temporal separation between the reflected and the refracted light of an individual particle thanks to a small probe volume formed by two strongly focused laser beams. It imposes that the diameter of the particle must be of the same order or greater than the 
beam waist diameter. But in this case, the measurement quality depends on the longitudinal location of the particles in the probe volume because of the strong curvature of the wave front.

To avoid the sensitivity to the wave front curvature of the laser beam, we introduce an alternative technique, called Glare Spot Phase Doppler Anemometry (GS-PDA). In this configuration, two large beams are used to form the probe volume and two detectors are located in the same plane as the incident beams, similar as in the planar PDA. But the detectors are in the image plane of the particle and in the forward direction at an angle where the intensities of the reflection and the first order refraction are about in the same order. So the reflected and refracted light by the particle is separated in space. When a particle passes through the probe volume, each detector obtains a signal composed of two separate parts, one due to the reflection and the other due to the refraction.

In order to comprehend out the characteristics of the GS-PDA and its sensibilities to particle size, refractive index, as well as velocity, a numerical study of this technique is presented in this paper by two complementary methods: 1). geometrical optics model (GOM) permitting to understand the principle and to treat the problem in a simple way and 2). full electromagnetic model (EIM) thanks to a simulation code of imaging for small particle ${ }^{[19]}$. Since the main purpose of the paper is to examine the principle of the PS-PDA and its sensibility to different parameters as particle size, refractive index and velocity, the study will be undertaken step by step for three configurations: 1). one beam - one detector, 2). two beams - one detector and 3). two beams - two detectors. To simplify the analysis, the form of the detectors has not taken into account, i.e. the intensity in the image plane is considered as measured signal.

The paper is organized as follows. A brief presentation of the physical origin and the properties of the glare spots will be given in section 2. Section 3 is devoted to the geometrical optics model of GS-PDA. The full electromagnetic imaging model will be introduced and 
applied in section 4 to the study of the measurement of the particle refractive index and diameter.

The conclusions and perspectives are given in the last section.

\section{Glare spots}

When a particle is illuminated by a beam of light, it scatters light in all space. If the particle is much larger than the wavelength of the incident beam we can observe distinctively in certain angle two bright spots on the particle surface, which are called glare spots ${ }^{[15]}$. The formation of the glare spots can be explained in a geometrical optics point of view due to the separation of reflected and refracted light.

We consider a particle of diameter $d$ and refractive index $m$ illuminated by a laser beam. The scattered light is collected by a lens of diameter $\phi$ and focal distance $f$ located in the direction $\theta$ relative to the incident beam (Fig. 1). Two bright points are then formed on the image plan: one ( $A^{\prime}$ in Fig. 1$)$ is created by the refracted light and the other $\left(R^{\prime}\right)$ by the reflected light.

When a particle is illuminated by two beams (similar to the Planar Phase Doppler Anemometry, but here the detector is in the image plane of the particle, see Fig. 2), the light due to the reflection and the refraction from the two beams interfere. When a particle passes through the probe volume, the signal obtained by a detector is composed of two Doppler-like bursts, one due to the reflection and the other to the refraction. The detailed analysis of such a configuration will be examined with the geometrical optics model (GOM) and the full electromagnetic imagine model (EIM) in the following sections.

\section{Geometrical optics model}

The geometrical optics is the most simple and instructive approach to understand the behaviour of GS-PDA. At the first stage, we do not take into consideration the lens. 
When a particle of refractive index $m$ and diameter $d$ is illuminated by a plane wave of wavelength $\lambda$, the glare spots' positions can be determined by the refracted and reflected rays (Fig. 3). The positions of the glare spots depend not only on the refractive index and the diameter of the particle, but also on the detection angle $\theta$.

The detector being far from the particle, we suppose here for the simplicity that the reflected and refracted rays are parallel.

For convenience, we define the reference ray as the line coming from the source at infinity, passing by the centre of the particle and going out in the direction of emerging rays. The distances between the reference ray and the reflected ray is noted $P_{r}$, and respectively $P_{a}$ for the refracted ray (the subscript $r$ and $a$ represent respectively reflection and refraction). $P_{r}$ and $P_{a}$ are then given by:

$$
P_{r}=\frac{d}{2} \cos \frac{\theta}{2}
$$

and

$$
P_{a}=\frac{d}{2} \cos \tau
$$

Since $2 \tau^{\prime}-2 \tau=\theta$, according to the Snell-Descartes law :

$$
m \cos \tau^{\prime}=\cos \tau
$$

$P_{a}$ can then be written as :

$$
P_{a}=\frac{d m \sin \left(\frac{\theta}{2}\right)}{2 \sqrt{1+m^{2}-2 m \cos \left(\frac{\theta}{2}\right)}}
$$

It is clear that the distance $P_{r}$ for the reflected ray does not depends on the refractive index but the distance $P_{a}$ does ${ }^{[16][17]}$. The distance between the two spots $D_{s}=P_{r}+P_{a}$ is proportional to the diameter and depends on the refractive index and the collection angle.

The intensities of the reflected and refracted light can be also calculated according to the geometrical optics. The reflected intensity is given by: 


$$
I_{a}=I_{0}\left(1-r_{i}^{2}\right)^{2} \frac{d^{2}}{l^{2}} \frac{m\left(1-m \cos (\theta / 2)^{2}\right.}{\left(m^{2}-1\right) \cos (\theta / 2)\left(1+m^{2}-2 m \cos (\theta / 2)\right.}
$$

where $I_{0}$ is the intensity of incident light, $r_{i}$ is the Fresnel reflection factor for parallel $(i=1)$ or perpendicular $(i=2)$ polarization, $l$ the distance between the particle and the observation point.

When the particle is illuminated by two beams with an angle $2 \alpha$ between them, the reflected and refracted light from the two beams interferes. The optical path difference for the reflected and refracted rays are respectively given by:

$$
\delta_{r}=d\left[\sin \left(\frac{\theta-\alpha}{2}\right)-\sin \left(\frac{\theta+\alpha}{2}\right)\right]
$$

for reflection and

$$
\delta_{a}=d\left[\sqrt{1+m^{2}-2 m \cos \left(\frac{\theta+\alpha}{2}\right)}-\sqrt{1+m^{2}-2 m \cos \left(\frac{\theta-\alpha}{2}\right)}\right]
$$

for refraction.

The corresponding phase differences for reflected and refracted light are then $\phi_{r}=\frac{2 \pi \delta_{r}}{\lambda}$ and $\phi_{a}=\frac{2 \pi \delta_{a}}{\lambda}$ respectively. They are both proportional to the particle diameter so they can be rewritten as $\phi_{r}=A_{r} d$ and $\phi_{a}=A_{a} d$. Note that the factor $A_{r}$ does not depends on the refractive index but $A_{a}$ does. The glare spot intensities on the image plane vary according to the particle size and are modulated by the phase differences.

Again to simplify the analysis, we suppose that the reflected intensities from the two beams are equal on the image plane, so does the refracted intensities. Then we can write:

$$
I_{r}=T_{r} d^{2} \cos ^{2}\left(\frac{A_{r} d}{2}\right) \quad I_{a}=T_{a} d^{2} \cos ^{2}\left(\frac{A_{a} d}{2}\right)
$$

where $T_{r}$ and $T_{a}$ are two coefficients depending on the reflection and refraction Fresnel coefficients and the optical configuration. 
The figure 4 shows the behaviour of the reflected and refracted light intensities from a water droplet $(m=1.33)$ illuminated by two planes waves with a half-angle $\alpha=5^{\circ}$, the collection angle is $\theta=66.5^{\circ}$. The two factors $A_{r}$ and $A_{a}$ are respectively equal to $A_{r}=0.299 \pi$ and $A_{a}=0.3533 \pi$. The intensity of the spots varies periodically with particle diameter. The corresponding periods of $d$ are then $6.689 \mu \mathrm{m}$ and $5.661 \mu \mathrm{m}$ for reflection and refraction respectively. It is worth to point out that if the intensities due to the two beams are not equal, the periods remain the same but the contrast will be worse.

This simple model of geometrical optics predicts the skeleton of behaviour of glare spots in the GS-PDA. The significant parameters as the lens dimension, the focal length, and the high order refraction effects have not be taken into account. These questions will be examined in the next section with a full electromagnetic model.

\section{Electromagnetic imaging model}

In this section an electromagnetic imaging model based on full solution of Maxell's equations and Huygens-Fresnel diffraction principle will be used. The model will be presented in the first subsection and it will then be applied to one or two beam(s) configurations of a GS-PDA system.

The electromagnetic imaging model (EIM) is based on the work of Ren et al. ${ }^{[19][20][21]}$. We consider a particle of diameter $d$ and refractive index $m$ located arbitrarily in a laser beam of wavelength $\lambda$. The calculation of the intensity in the image plane consists of three steps. The scattered field on the surface of the lens is firstly calculated by the Lorenz-Mie theory or generalised Lorenz-Mie theory ${ }^{[22]}$. The role of the lens is then taken into account by the thin lens transformation $^{[23]}$. The intensity on the detector is finally calculated by Huygens-Fresnel integration $^{[24][25][26]}$ of the electromagnetic field on exit surface the lens. 


\subsection{Glare spots of a particle illuminated by one beam}

When a particle is illuminated by one beam, two glare spots are formed on the image plane (see Fig. 1). Their intensities and the distance between them depend on the properties of droplets (size and refractive index) and the optical configuration.

We consider a water droplet illuminated by a plane wave of wavelength $0.488 \mu \mathrm{m}$. The detector is located at $\theta=66.875^{\circ}$, in the image plane, $400 \mathrm{~mm}$ from the lens. The diameter and the focal length of the collection lens are respectively $15 \mathrm{~mm}$ and $200 \mathrm{~mm}$. The magnification of this configuration is then equal to 1 . The collection angle of the lens is then $\Delta \theta=1.074^{\circ}$.

Firstly, we compare the evolutions of the maximum intensities in the glare spots predicted by the GOM and EIM. Fig. 5 presents the evolution of the intensities as function of the particle diameter for refractive index $m=1.33$. The incident beam is polarized perpendicularly to the scattering plane. We note that the mean intensity of reflection of EIM agrees well with that of GOM but the refraction intensity of EIM is more important than that of GOM. The predictions of EIM present strong fluctuations for both reflection and refraction because in the simulation of EIM all the scattered light have been taken into account while in the GOM only the reflection or the first order refraction is considered. At the chosen angle, the amplitudes of reflected and refracted light dominate and higher orders are relatively small so they contribute mainly to the oscillation. It is worth to note that the relative ratio between reflected and refracted intensities in Figs. 4 and 5 are different because in one beam configuration (Fig. 5), the scattering angle is $66.8^{\circ}$ whereas for the two beam configuration (Fig. 4) the collection angles relative to the two incident beams are respectively $66.5+5^{\circ}$ and $66.5-5^{\circ}$.

According to the geometrical optics, the reflected and refracted intensity ratio has a simple relation with the refractive index, potentially permitting the measurement of the refractive index. Schaller and al ${ }^{[27][28]}$ have examined this relation with geometrical optics and Lorenz-Mie theory and by experiments. They found strong fluctuation of the intensity ratio as function of the 
refractive index and the particle size. We have also examined this relation by the EIM with full GLMT (Generalized Lorenz-Mie Theory). Fig. 6 presents the intensity ratio of reflected and refracted intensities versus the refractive index for a particle of diameter $50 \mu \mathrm{m}$ or $100 \mu \mathrm{m}$, illuminated by a plane wave $(\lambda=0.488 \mu \mathrm{m})$. We find that even the tendency of the variation predicted by EIM and GOM is the similar : the intensity ratio increases as function of refractive index, the discrepancy is significant and the result of EIM is somewhat "chaotic", especially for a small particle.

Then, the distance between the two spots predicted by EIM and by GOM are compared. This distance depends both on the particle diameter and the refractive index. Fig. 7 shows the distance between them for a particle of refractive index 1.33 . We find that when the diameter is greater than a critic value, here of $20 \mu \mathrm{m}$, the distance is proportional to the particle diameter and the agreement between EIM and GOM is good, the deviation is less than $10 \%$. The simulations have also been done for a magnification of 2 and a collection angle $\Delta \theta=1.432^{\circ}$. We found that the under limit of the particle diameter is practically independent of the magnification but depends on the collection angle. When the collection angle $\Delta \theta$ is $1.432^{\circ}$ the under limit is pushed to $15 \mu \mathrm{m}$ for both magnifications of 1 and 2 . This effect is due mainly to the diffraction of the lens. When the lens is sufficiently large this effect will be negligible. So the distance between the spots can be used to determine the diameter of the particle.

When a particle passes through the probe volume created by two beams, two Doppler-like bursts are obtained by each detector since in one hand, the scattered light from the beams interfere to create the "fringes", on the other hand, each detector located on the image plane of the particle "sees" subsequently the reflected and refracted glair spots. Hess et al have used the pulse displacement (or time delay) to measure the diameter of the particle and they supposed that the velocity of the particle is perpendicular to the fringes ${ }^{[28]}$. We will show in the section 4.3 that two beam - one detector configuration permits to measure simultaneously $x$ component of the 
velocity $V_{\mathrm{x}}$ and the diameter of the particle $D$. If two detectors are used, the $V_{\mathrm{x}}$ and $D$ can be measured respectively by the frequencies and the phase differences and $y$ component of the velocity can be determined by the time delay between the two maxima of the pedestal which correspond to the two glare spots of reflection and refraction.

\subsection{Glare spots of a particle illuminated by two beams}

When the particle is illuminated by two beams, the total intensity on the detector is the result of the sum of the electric fields scattered by the particle from the two beams. The two scattered fields then interfere on the detector. We examine in this section the characteristics of the glare spots obtained when a particle is illuminated by two large beams. The configuration considered is shown in Fig. 2. The lens axis makes an angle of $\theta=66.5^{\circ}$ relative to the bisect of the two beams of a semi-angle $\alpha=5^{\circ}$ between them. The scattering angles are then $61.5^{\circ}$ and $71.5^{\circ}$. The particle moves in the direction perpendicularly to the bisect of the two beams.

To show the interference of the light scattered from the two beams, we examine now the intensity in the image plane when the particle is illuminated by only the beam 1 or beam 2 , or by the two beams. Fig. 8 shows the intensities on image plane when a water droplet $(m=1.33)$ of diameter $50 \mu \mathrm{m}$ is illuminated by one beam or by two beams. The glare spots due to the reflection (right) and refraction(left) are well separated and their positions correspond to the prediction of geometrical optics. The glare spot due to the reflection is at $20.5 \mu \mathrm{m}$ for one beam and at $22.0 \mu \mathrm{m}$ for the other because the two beams are not symmetrical about the detector. The glare spots due to the refraction is at $-24.5 \mu \mathrm{m}$.

We note that the intensities of reflected and refracted glare spots in one beam case $\left(\right.$ at $71.5^{\circ}$ or $61.5^{\circ}$ ) are very different. The intensity ratios of reflected and refracted glare spots are 0.7 at for $71.5^{\circ}$ and 2.48 for $61.5^{\circ}$. The total intensity of the two beams in the glare spot due to the reflection is smaller than that of one beam illumination, the electromagnetic fields from the two 
beams are destructive. The total intensity of the glare spot due to the refraction is greater than that one beam illumination, the fields scattered from the two beams are constructive.

In fact, these interferences (construction or destruction) depend also on the particle diameter. Fig. 9 shows the total intensity versus the diameter. The profiles of the intensity evolution of the glare spots are periodical in function of the particle diameter as predicted by geometrical optics (Fig. 4) and the period corresponds well to that of geometrical optics. But it is worth noting that minimum intensity is not zero and increases with the particle diameter. There exists also a high frequency fluctuation. These can be explained by the fact that the size of the lens is finite (15 mm for this simulation) and all orders of scattered light is taken into account in EIM.

\subsection{Glare spots Phase Doppler Anemometry}

If the detector is small enough relative to the size of the spots, when a particle passes through the probe volume, the two spots' images pass successively in the detector and the detector "sees" the reflection and refraction glair spots one after the other, we then obtain a signal composed of two Doppler-like bursts. A typical GS-PDA signal simulated by EIM is presented in Fig. 10. A particle of $80 \mu \mathrm{m}$ passes along $x$ axis through the probe volume formed by two plane waves of wavelength $0.488 \mu \mathrm{m}$ with a half angle of $5^{\circ}$ between them (Fig. 2). This signal is similar to the signals obtained by a dual burst PDA in which two tightly focused beams are used $^{[11][12]}$.

The fringe space in this configuration is equal to $\delta=2.8 \mu \mathrm{m}$. The velocity of the particle is assumed to be $1.0 \mathrm{~m} / \mathrm{s}$ and the period of the signal is then $2.8 \mu \mathrm{s}$. The two parts due to the reflected and refracted light are well distinct in the signal. By the Fourier transformation we obtained the main frequency of the signal $f=0.357 \mathrm{MHz}$. We can also obtained the pedestals of the signal by filtering the high frequency and extract the distance between the two spots. 
It is worth noting that when a particle is illuminated by two beams and one detector is used, there exist specific positions for which the reflection and/or refraction spots disappear as shown in figures 4, 8 and 9. In this case no distance between the two spots can be extracted. But when the particle moves in the probe volume we can always obtain a Doppler-like signal to extract the pedestals even for some positions the intensity is destructives, for example the point A for refraction and B for reflection in Fig. 10.

We investigate in the following the distance between the two maxima of pedestal as function of the particle size. For the simulation presented in Fig. 10, the diameter is $80 \mu \mathrm{m}$ and the refractive index $m=1.33$. The detector is located at $\theta=67.574^{\circ}$ so that the reflected and refracted light is of the same order of intensity.

Firstly, we note that the $x$ component of velocity can be determined by the frequency of the signals:

$$
V_{x}=f \delta=0.357 \times 10^{6} \times 2.8 \times 10^{-6}=1.0 \mathrm{~m} / \mathrm{s}
$$

The time delay between the two maxima in the pedestal (dashed line in Fig. 10) $T_{\mathrm{s}}$ is related then to the displacement of the particle $D_{x}$ and the distance between the spots $D_{s}$ by:

$$
D_{s}=g D_{x} \cos \theta=g V_{x} T_{s} \cos \theta
$$

where $g$ is the magnification of the used optics. Since the particle diameter $d$ is related to $D_{s}=P_{a}+P_{r}$ (see Eqs. (1) and (4)), the particle diameter can then be obtained from the time delay between the two maxima in the pedestal $T_{s}$.

For the case shown in Fig. 10, the time delay between the two maxima in the pedestal is $T_{\mathrm{s}}=192 \mu \mathrm{s}$ which corresponds to a displacement of $D_{x}=192 \mu \mathrm{m}$. Considering the angular position of the detector, the distance of between the two spots $D_{\mathrm{s}}=72.6 \mu \mathrm{m}$ can then be deduced and it is in agreement with that predicted in Fig. 7.

Then the simulation has been done for a water droplet of diameter from $30 \mu \mathrm{m}$ to $120 \mu \mathrm{m}$ and the result is presented in Fig. 11. We found that the relation $D_{\mathrm{s}}-d$ is linear and the agreement 
between EIM and GOM is very good. Therefore the distance $D_{s}$ permits to determine the diameter of the particle.

On the other hand, since both the position of the refraction spot and the ratio of the two maximum intensities depend also on the refractive index, we would expect to obtain information about refractive index from these values if the diameter is known. But both GOM and EIM show that the distances $D_{s}$ depends little on the refractive index. Furthermore, the intensity ratio obtained from EIM fluctuates as function of the refractive index and the fluctuation is too strong to be used to determine the refractive index of an individual particle. We will show in the following section that the two beams - two detectors configuration permits to get better information of the refractive index.

Now we add a second lens and a detector same as the first but with a different collection angle. The lens and the detectors are all in the same plane as the incident beams. In this configuration, when a particle passes through the probe volume, two signals will be obtained as in the dual burst $\mathrm{PDA}^{[11][12]}$, each of them is composed of two parts: one due to reflection and the other due to refraction. Besides the distances $D_{\mathrm{s}}$ and intensity ratios as described above, we can also extract the two phase differences between the two signals separately for reflection and refraction parts. Fig. 12 shows two signals simulated by EIM. The two detectors are located in $65.426^{\circ}$ and $67.574^{\circ}$ relative to the bisect of the two beams. Firstly, we remark that the distances between the two intensity maxima are not the same because the two detectors are not symmetric about the particle trajectory. Note that, in principle, the relation between the phase difference and the particle diameter, and that between the frequency and the transverse velocity component remain the same as in Planar PDA if the particle is located in the object plane.

The phase differences extracted from the signals are shown in Fig. 13. We find that the phase differences are relatively stable around the maximum intensity (it should be noted that the phase difference for signals of very weak intensity has no sense since the noise can be more 
important than the signals). The average phase differences for reflection and refraction are respectively $43^{\circ}$ and $24^{\circ}$. The same simulation has been done for different particle size and refractive index. The results are presented in Figs.14 and 15. The evolution tendency of the phase differences predicted by EIM agrees with those predicted by geometrical optics. Larger is the diameter, better is the agreement. But the evolution of phase differences with the diameter and the refractive index predicted by EIM are not linear( chaotic in certain sense) as predicted by GOM. Nevertheless we find that when the particle is very large (lager than $100 \mu \mathrm{m}$ ), the phase differences can be used to determined the diameter with the precision about $30 \%$ (still too important, see comments in the last section).

Nevertheless, the mean value of the ratio of the two phase differences obtained by EIM is relatively stable. Left part of the Fig. 16 shows the ratio predicted by EIM and GOM for a particle of diameter from $25 \mu \mathrm{m}$ to $150 \mu \mathrm{m}$ and refractive index 1.33 . The mean value of the ratio predicted by EIM is in agreement with that of GOM. Therefore the mean value of the refractive index can be determined according to the relation of OGM (right part of the figure).

Once the particle diameter is determined by the two phase differences, the two time delays $\Delta_{i}$ between the two maxima of the pedestals obtained from the signals of the two detectors can be used to determine $y$ component of the velocity in the plane of the beams according to:

$$
V_{z}=\frac{D_{s}}{\Delta_{i} \sin \theta_{i}}-\frac{V_{x}}{\tan \theta_{i}}
$$

where $i=1,2$ corresponds to the detector 1 or $2, D_{s}=P_{r}+P_{a}$ is the distance between the two glare spots predicted by GOM. $V_{x}$ is the $x$ component of the velocity perpendicular to the fringes determined by the frequency of the signal.

\section{Conclusions and perspectives}

A new technique, Glare Spots Phase Doppler Anemometry, is proposed and studied by using the geometrical optics model and the full electromagnetic imaging model. The simulations 
by EIM agree in general with those predicted by GOM and shows that the GS-PDA provides riche information: two distances and two intensity ratios between the reflection and refraction Doppler-like bursts, two phase differences as well as the frequency of the signals. The particle diameter and two components of velocity can be determined by the time delays between the two bursts, the phase differences and the frequency of the two signals. The refractive index can be evaluated from the mean value of the phase difference ratios of the two signals.

Nevertheless, it should be pointed out that the relations of the refractive index with the phase difference and the intensity ratio obtained by the simulation for individual particles are somewhat "chaotic" (even the mean value has sense). Further study must be carried out to determine if it is the intrinsic property of the glare spots or due to the diffraction effect since the diameter of the lens is only $15 \mathrm{~mm}$ in the simulation because of numerical difficulty. Two possible ways could be involved : check by experiment with realistic lens and detectors or simulation by improved code permitting to take into account the effect of the size of the lens, the form of the detectors and others parameters.

\section{Acknowledgements}

This work is supported partially by the inciting joint action, ACI "Non-pollution, dépollution", VITAMA, project 47. This ACI is financed by the FNS, CNRS and the DGA of France.

\section{References}

[1] J. P. A. J. van Beeck and M. L. Riethmuller, "Rainbow phenomena applied to the measurement of droplet size and velocity and to the detection of non-sphericity", Applied Optics, pp2259-2266, 1996

[2] J. P. A. J. van Beek, D. Giannouslis, L. Zimmer and M. L. Riethmuller, "Global rainbow thermometry for droplet temperature measurement", Optics letters, 24(23), 1696-1698, 1999

[3] J. P. A. van Beeck , T. Grosges and M.G. De Giorgi, "Global rainbow thermometry assessed by Airy and Lorenz-Mie theories and compared with phase Doppler anemometry", App. Opt. 42(19): 4016-4022, 2003. 
[4] P. Lavieille, F. Lemoine, G. Laverge, JF Virepinte, M. Lebouché, "Temperature measurements on droplets in monodisperse stream using laser-induced fluorescence", Exp. Fluids, 29: 429-437, 2000.

[5] J. Sakakibara, R. J. Adrian, "Whole field measurement of temperature in water using twocolor laser-induced fluorescence", Exp. Fluids, 26:7-15, 1999

[6] P. Lavieille, F. Lemoine, G. Laverge, JF Virepinte, M. Lebouché, "Evaperating and combusting droplet temperature measurements using two-color laser-induced fluorescence", Exp. Fluids, 31:45-55, 2001.

[7] H. E. Albrecht, N. Damaschke, M. Borys and C. Tropea, Laser Doppler and Phase Doppler Measurement Techniques, Berlin: Springer, 2003

[8] G. Brenn and F. Durst, "Refractive index measurements using the phase-Doppler technique", Atomization and Sprays, 5, 545-567, 1995

[9] A. Naqwi, F. Durst, and X. Liu, "Extended Phase-Doppler System for Characterization of Multiphase Flows", Part. Part. Syst. Charact., 8(33), 16-22, 1991

[10]C. Tropea, T-H. Xu, F. Onofri, G. Gréhan, P. Haugen, Dual-Mode Phase Doppler Anemometer, Part. and Part. Syst. Charact. 13:165-170 (1996).

[11]F. Onofri, T. Girasole, G. Gréhan, G. Gouesbet, G. Brenn, J. Domnick, T. H. Xu, C.Tropea, "Phase-Doppler Anemometry with the Dual Burst Technique for Measurement of refractive index and Absorption Coefficient Simultaneously with size and Velocity", Part. Part. Syst. Charact., Vol 13, pp112-124, 1996

[12]F. Onofri, Prise en compte de la dimension finie des faisceaux d'éclairage en granulométrie optique : Anémométrie Phase Doppler-Diagnostics des milieux diphasiques. Ph. D Thesis, Rouen University, France, 1995

[13] S. V. Sankar, K. M. Ibbrahim, D.H. Buermann, M. J. Fidreich and W.D Bachalo, “An Integrated phase Doppler/rainbow Refractometer System for Simultaneous Measurement of Droplet Size, Velocity, and Refractive Index", Proc. $3^{\text {rd }}$ International Congress on Optical Particle Sizing, pp 275 284, Yokohoma, August 22-26, 1993

[14]A. Delconte, D. Blondel and F. Lemoine, Measurement of droplets size-temperature correlations in spray with combined 3 color LIF and PDA technique; ICLASS, Aug.27Sept.1, 2006, Kyoto, Japan

[15]H. C. Van de Hulst and R. T. Wang, "Glare points", Applied optics, 30(33), 4755-4763, 1991

[16]F. Durst, G Brenn and T. H. Xu, "A review of the development and characteristics of planar phase-Doppler anemometry". Meas. Sci. Tech. Vol 8 pp 1203-1221, 1997

[17] S. Dehaeck, J.P.A.J. van Beeck1and M.L. Riethmuller, "Glare Point Velocimetry and Sizing (GPVS) Introduction of a new optical 2D measuring technique for bubbly flows", 12th Int. Sym. Appl. Laser Tech. Fluid Mechanics, paper 11.4, Lisbon, 12-14 July 2004

[18]H. C. van de Hulst, Light Scattering by Small Particles, Dover Publications Inc., New York (1981).

[19]K. F. Ren, Diffusion des faisceaux feuille laser par une particule sphérique, $\mathrm{Ph}$. D. thesis, University of Rouen, France, pp167-190 , 1995

[20]K. F. Ren, T. Girasole, A. Taylor, G. Gouesbet, G. Gréhan, "Theoretical evaluation of a shadow Doppler velocimeter", Optics Communications, 220, 269-280, 2003 
[21]K. F. Ren, D. Lebrun, C. Ozkul, A. Kleitz, G. Gouesbet and G. Grehan, "On the measurements of particles by imaging methods : theoretical and experimental aspects", Part. Part. Syst. Charact., 13, pp. 156-164, 1996

[22] G. Gouesbet, B. Maheu, G. Gréhan, "Light scattering from a sphere arbitrarily located in a Gaussian beam, using a Bromwich formulation", J. Opt. Soc. Am. A 5(9), 1427-1443, 1988

[23] J.W. Goodman, Introduction à l'optique de Fourier et à l'holographie, Masson and Cie, Paris, 1972

[24]H. H. Hopkins, "The numerical evaluation of the frequency response of optical systems", Proc. Phys. Soc. B, 70:1002-1005, 1957

[25] J. J. Stamnes. Wave in Focal Regions. Adam Hilger, 1986

[26] S. A. Schaub, D. R. Alexander, J. P. Barton, "Theoretical model of laser imaging of small aerosols : applications to aerosol sizing”, Applied optics, Vol. 30, N³3, pp4777-4783, 1991

[27]J. K. Schaller, S. Wassenberg, D. K. Fiedler, C. G. Stajanoff, "A new method for temperature measurements of droplets”, Proc. ICLASS, Rouen pp443-450,1994

[28]J. K Schaller, S. Wassenberg, D.K Fiedler, C.G Stajanoff, "Refractive Index determination as a tool for temperature measurements and process control : a new approach", Proc SPIE, "Optical Measurements and sensors for the process Industries" Vol. 2248, pp318-324, 1994

[29] C.F. Hess C. Wood, "Pulse Displacement Technique to measure a single particle counter with a size range larger than 1000:1", Part. Part. Syst. Charact. Vol. 11, pp107-1013, 1994 


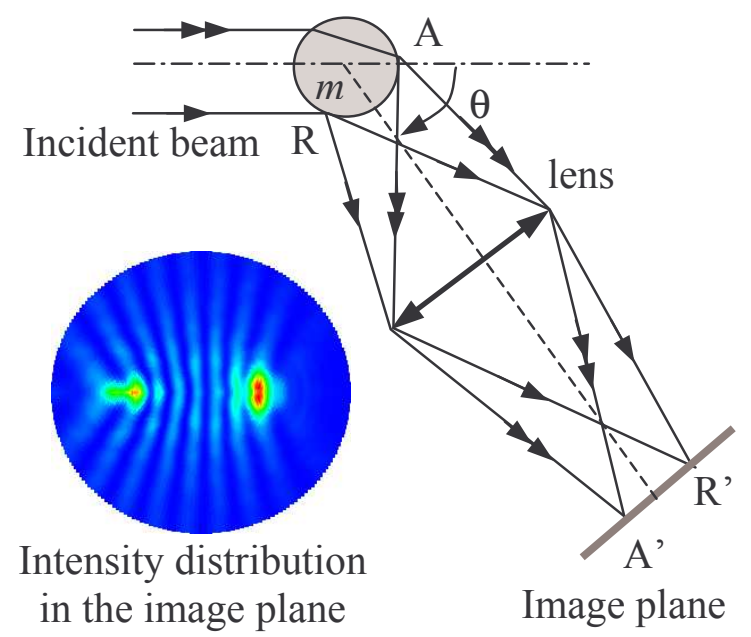

Fig. 1. Off-axis imaging. $A$ ' and $R$ ' are respectively the glair spots of refraction and reflection (colour on line). 
1

2

3

4

5

7

8

9

10

11

12

13

14

15

16

17

18

19

20

21

22

23

24

25

26

27

28

29

30

31

32

33

34

35

36

37

38

39

40

41

42

43

44

45

46

47

48

49

50

51

52

53

54

55

56

57

58

59

60

61

62

63

64

65

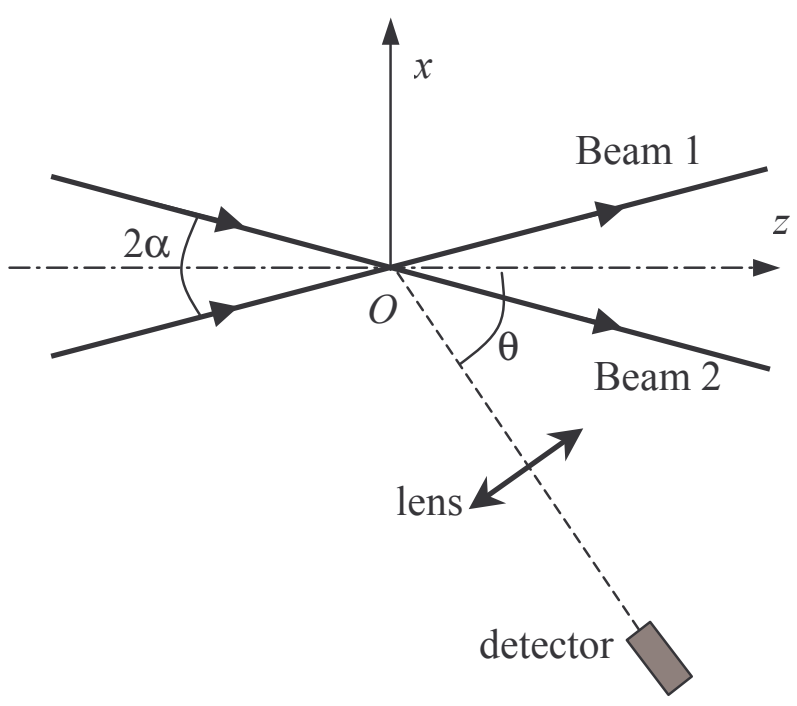

Fig. 2. Schema of a planar GS-PDA. The two incident beams, the lens and the detector are all in the same plane. The detector is located in the image plane of the particle at an angle where the reflection and first order refraction light intensities are in the same order. 
1

2

3

4

5

6

7

9

10

11

12

13

14

15

16

17

18

19

20

21

22

23

24

25

26

27

28

29

30

31

32

33

34

35

36

37

38

39

40

41

42

43

44

45

46

47

48

49

50

51

52

53

54

55

56

57

58

59

60

61

62

63

64

65

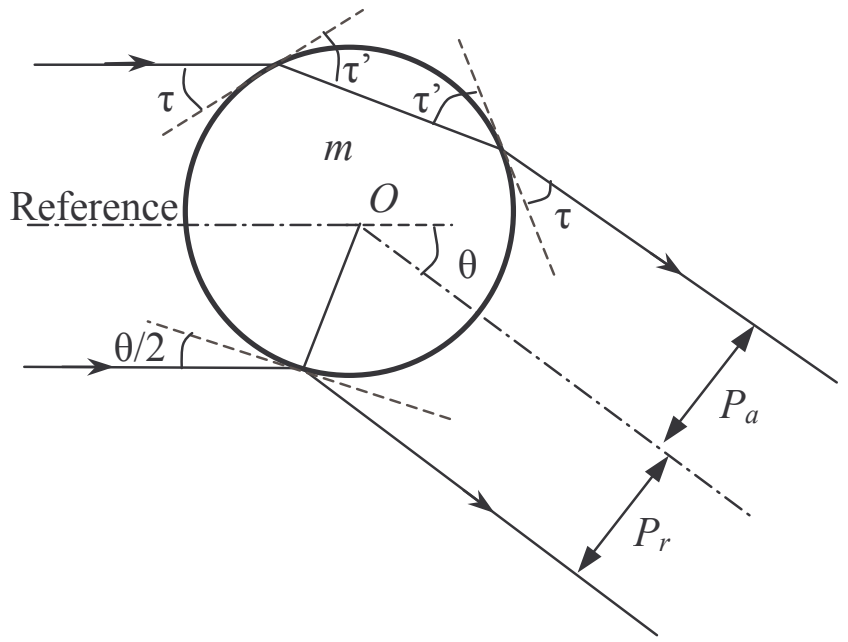

Fig. 3. Optical paths of a reflection ray and a refraction ray. 


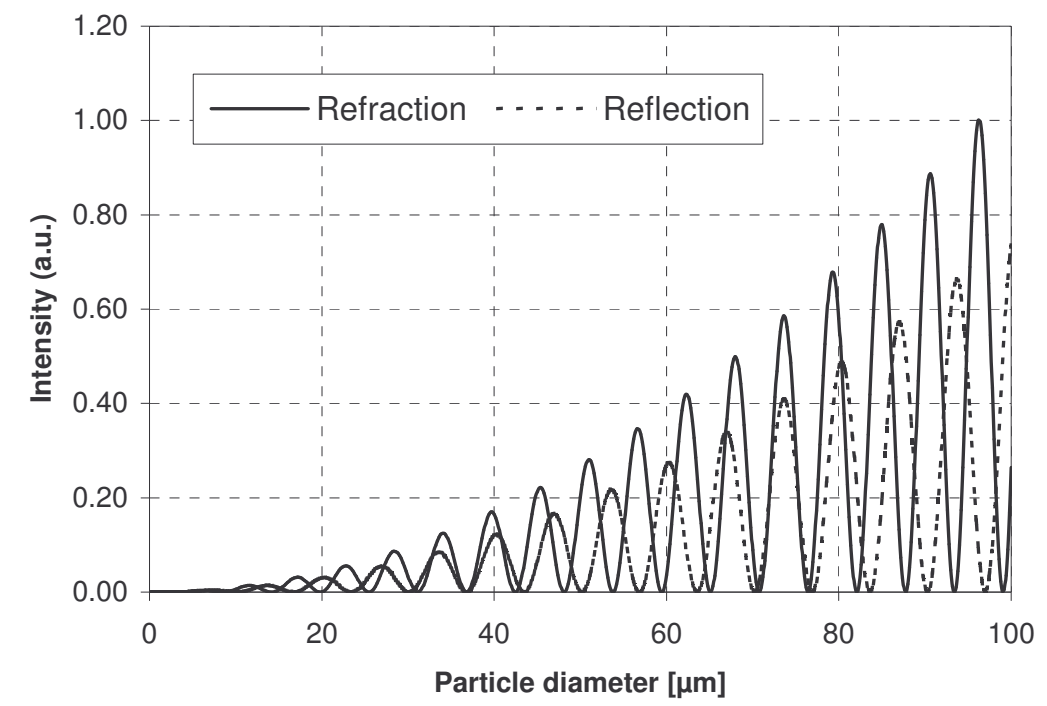

Fig. 4. Glare spot intensities as function of the particle diameter predicted by geometrical optics: a) for reflected light, b) for refracted light (the ratio of the reflected and the refracted intensities has been justified according to the Debye series calculation). 
1

2

3

4

5

6

7

8

9

10

11

12

13

14

15

16

17

18

19

20

21

22

23

24

25

26

27

28

29

30

31

32

33

34

35

36

37

38

39

40

41

42

43

44

45

46

47

48

49

50

51

52

53

54

55

56

57

58

59

60

61

62

63

64

65

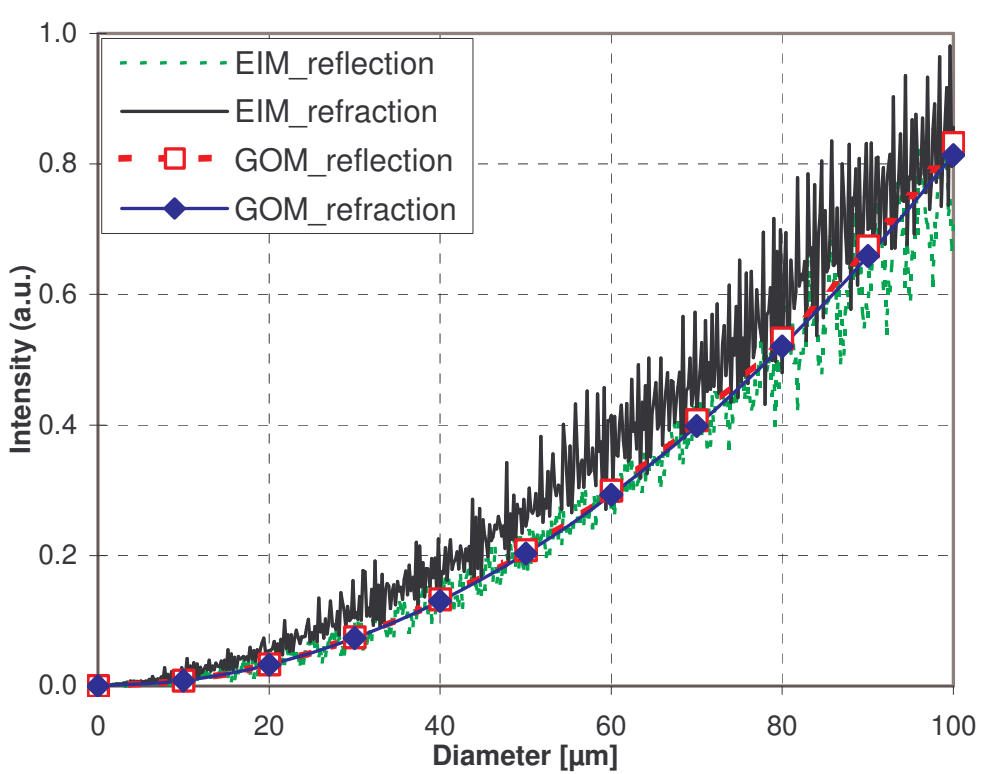

Fig. 5. Evolution of intensities of the glare spots versus the diameter of a water droplet ( $m=1.33)$ illuminated by a plane wave of wavelength $\lambda=0.488 \mu \mathrm{m}$ oriented at $66.785^{\circ}$ relative to the lens axis (colour on line). 
1

2

3

4

5

7

8

10

11

12

13

14

15

16

17

18

19

20

21

22

23

24

25

26

27

28

29

30

31

32

33

34

35

36

37

38

39

40

41

42

43

44

45

46

47

48

49

50

51

52

53

54

55

56

57

58

59

60

61

62

63

64

65

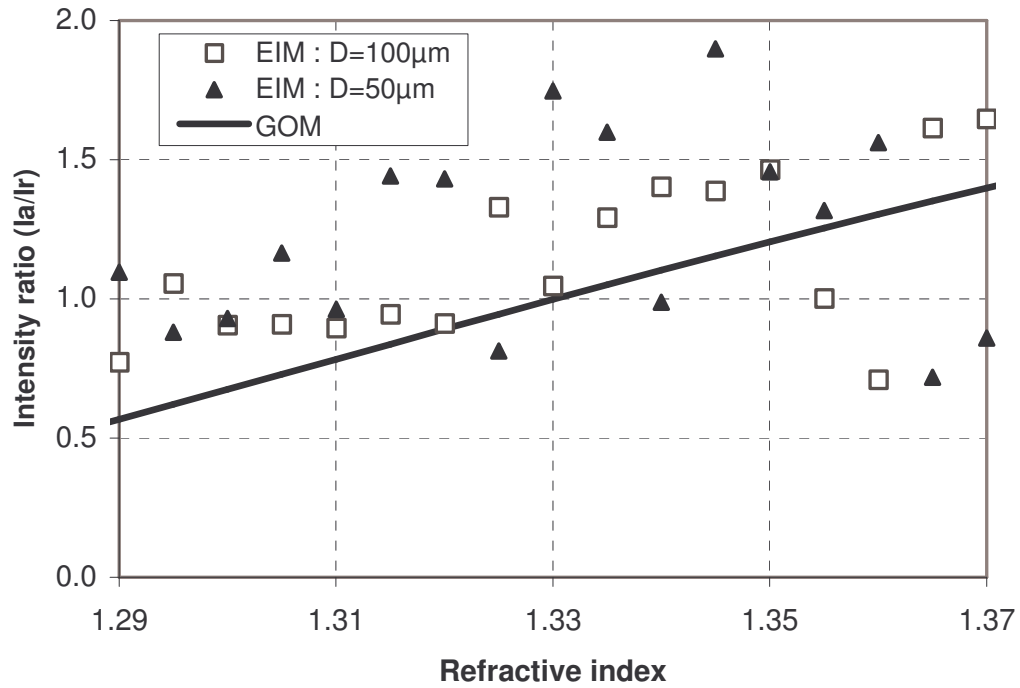

Fig. 6. Intensity ratios of the spots as function of the refractive index. 
1

2

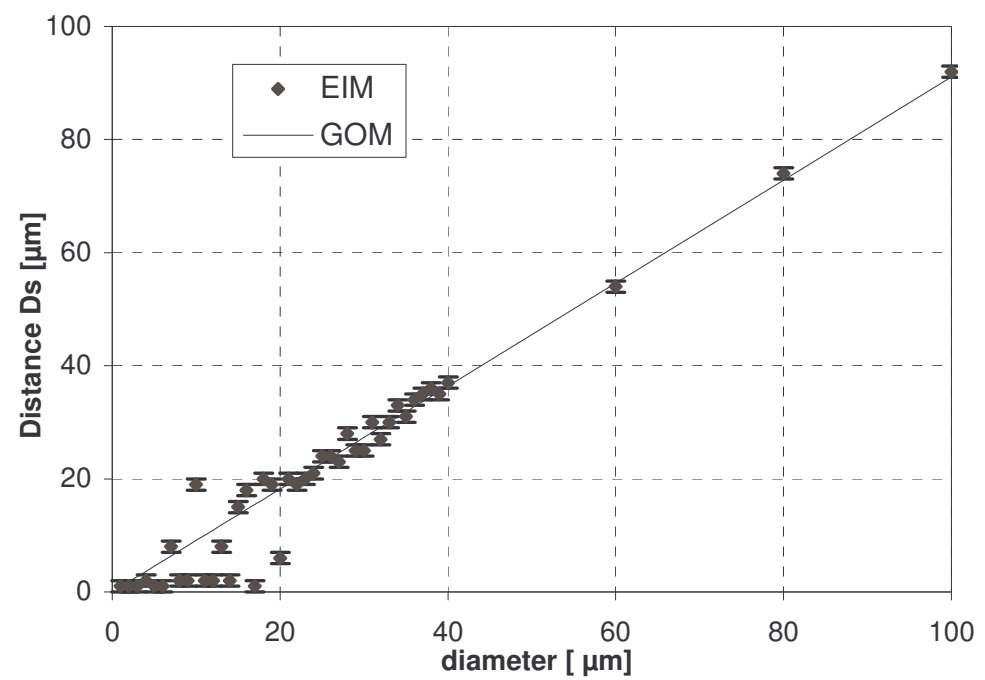

Fig. 7. Evolution of the spots' distance as function of the particle diameter. The refractive index is 1.33. 
1

2

3

4

5

6

7

8

9

10

11

12

13

14

15

16

17

18

19

20

21

22

23

24

25

26

27

28

29

30

31

32

33

34

35

36

37

38

39

40

41

42

43

44

45

46

47

48

49

50

51

52

53

54

55

56

57

58

59

60

61

62

63

64

65

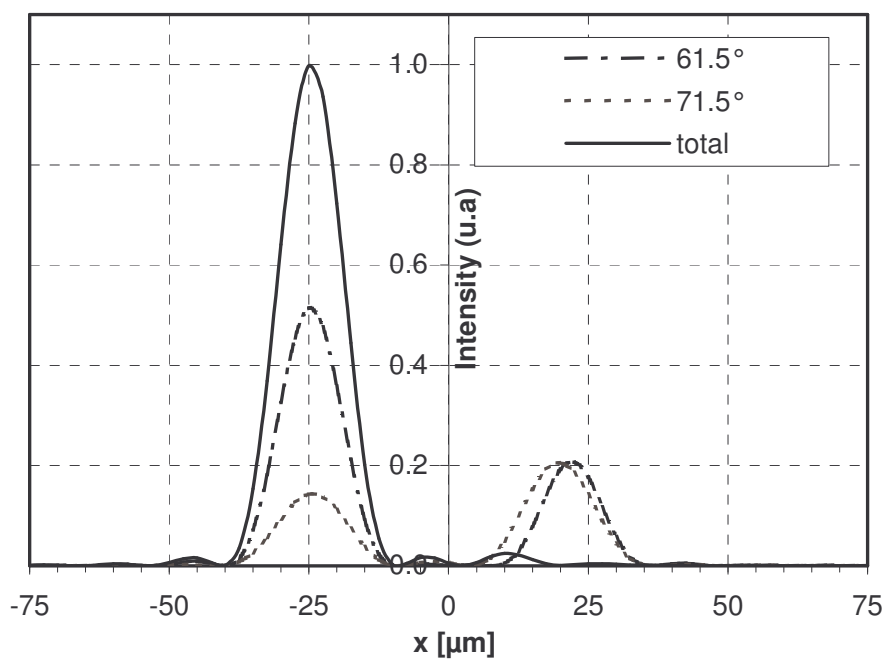

Fig. 8. Intensities on the image plane when a water droplet $(m=1.33, d=50 \mu m)$ is illuminated by one beam at $\theta=61.5^{\circ}$ (dashed line) or $\theta=71.5^{\circ}$ (long dashed line) or by two beams (solid line). 
1

2

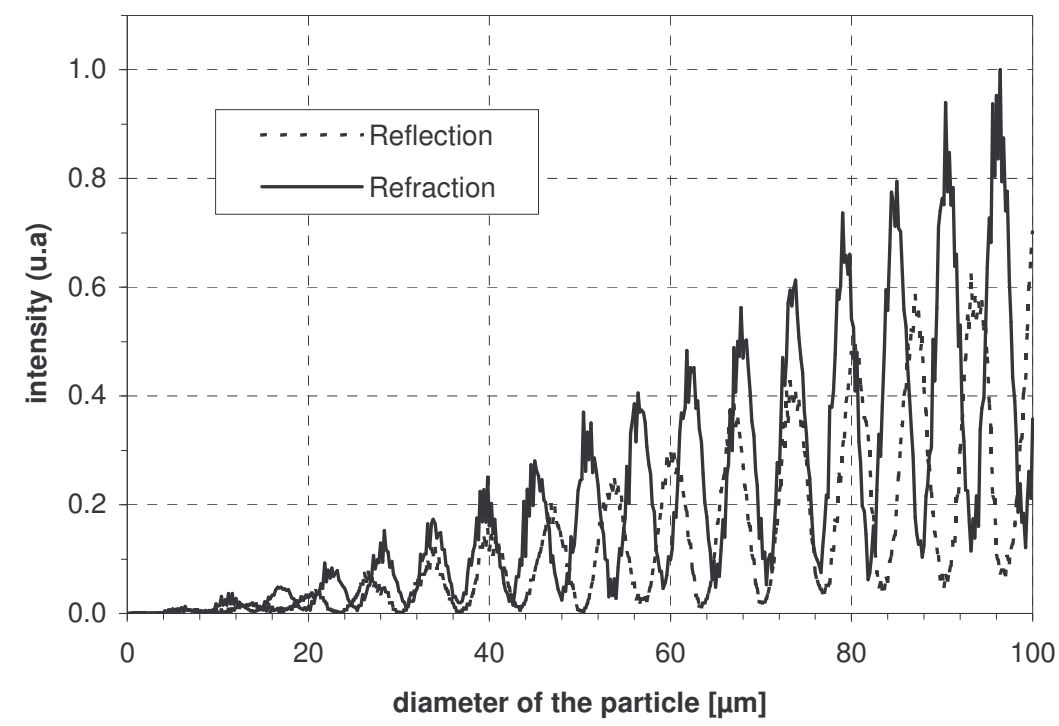

Fig. 9. Evolution of the glare spot intensities as function of the diameter of a water droplet illuminated by two plane waves. 


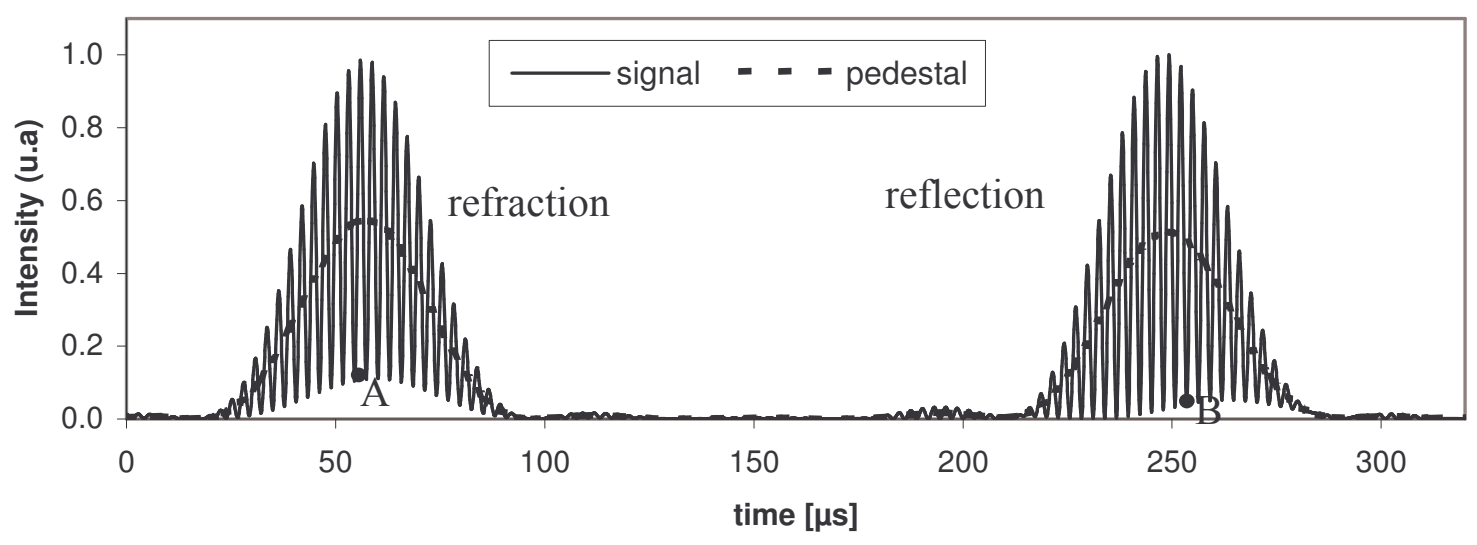

Fig. 10. Typical signal of GS-PDA. 
1

2

3

4

5

7

8

10

11

12

13

14

15

16

17

18

19

20

21

22

23

24

25

26

27

28

29

30

31

32

33

34

35

36

37

38

39

40

41

42

43

44

45

46

47

48

49

50

51

52

53

54

55

56

57

58

59

60

61

62

63

64

65

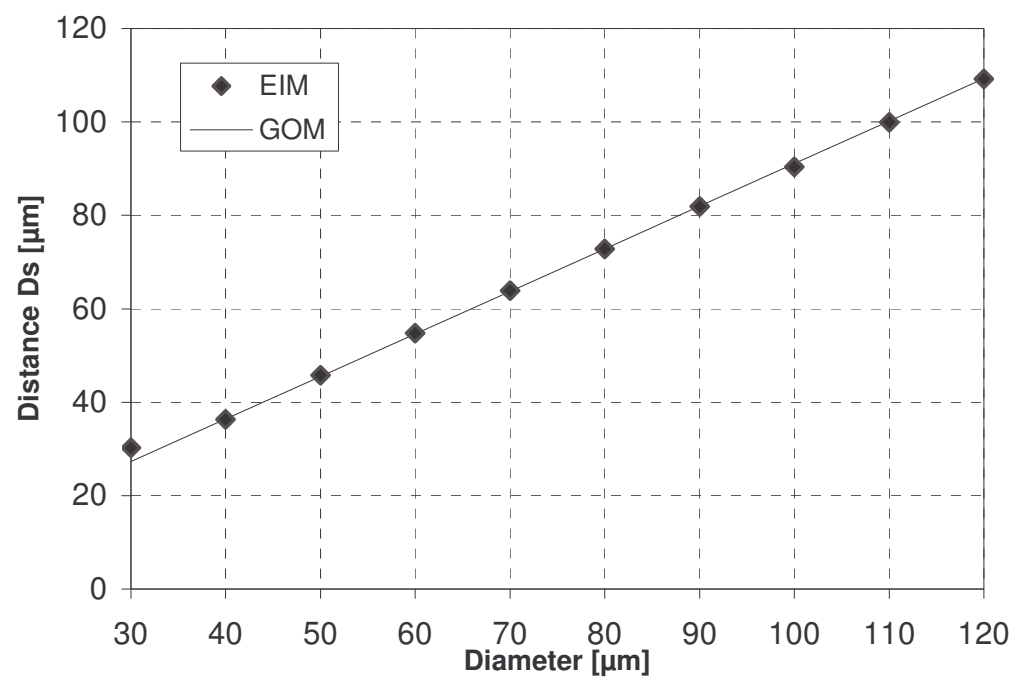

Fig. 11. Distance between the spots as function of diameter of a water droplet deduced from a GS-PDA signal. 
1

2

Fig. 12. Signals of GS-PDA obtained by two detectors located respectively at $\theta=65.426^{\circ}$ and $67.574^{\circ}$ with a half angle of $5^{\circ}$. A water droplet $(m=1.33, d=80 \mu \mathrm{m})$ is illuminated by two plane wave.

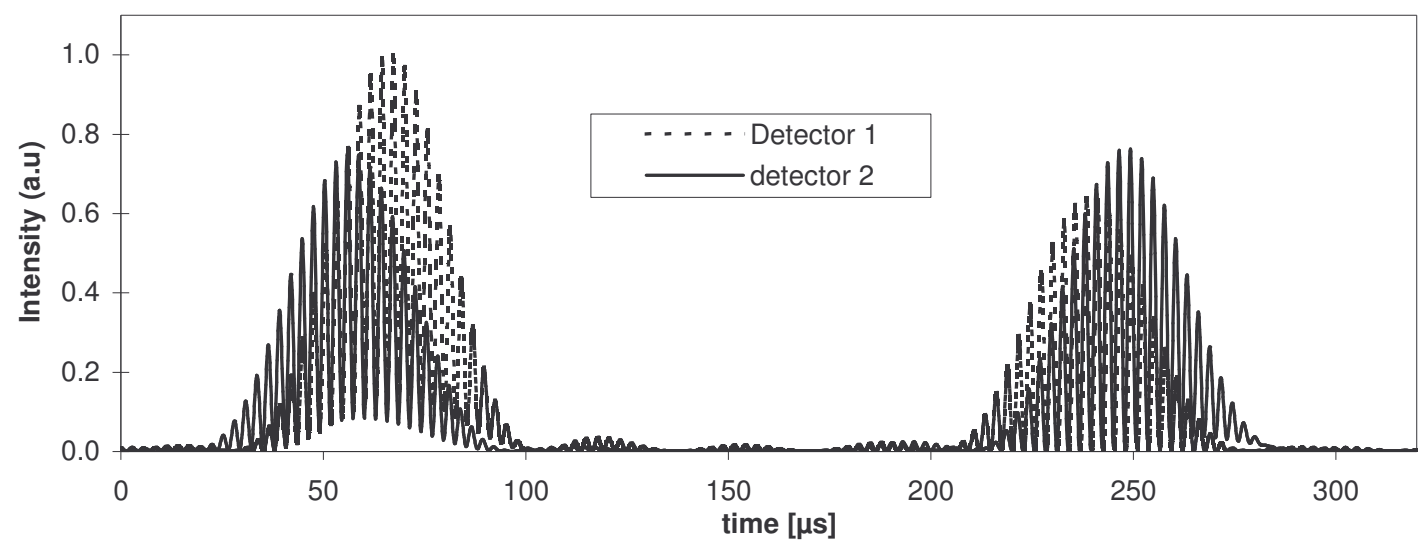


1

2

3

4

5

6

7

8

9
10

11

12

13

14

15

16

17

18

19

20

21

22

23

24

25

26

27

28

29

30

31

32

33

34

35

36

37

38

39

40

41

42

43

44

45

46

47

48

49

50

51

52

53

54

55

56

57

58

59

60

61

62

63

64

65

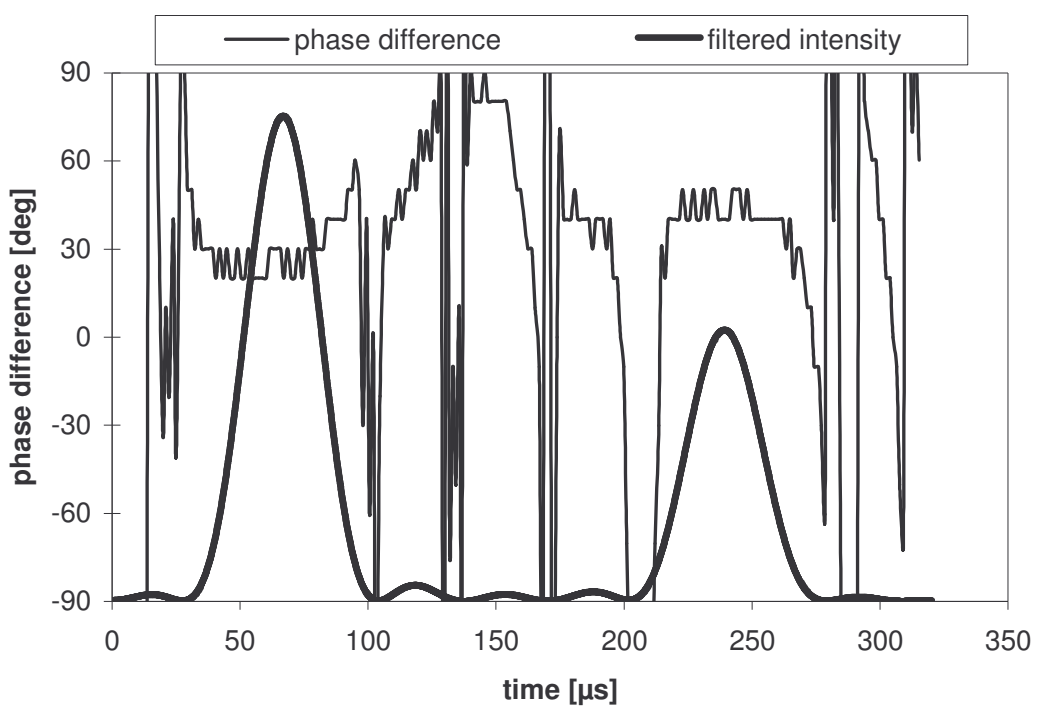

Fig. 13. Phase difference between the two signals and their pedestal obtained from the signals in Fig. 12. 


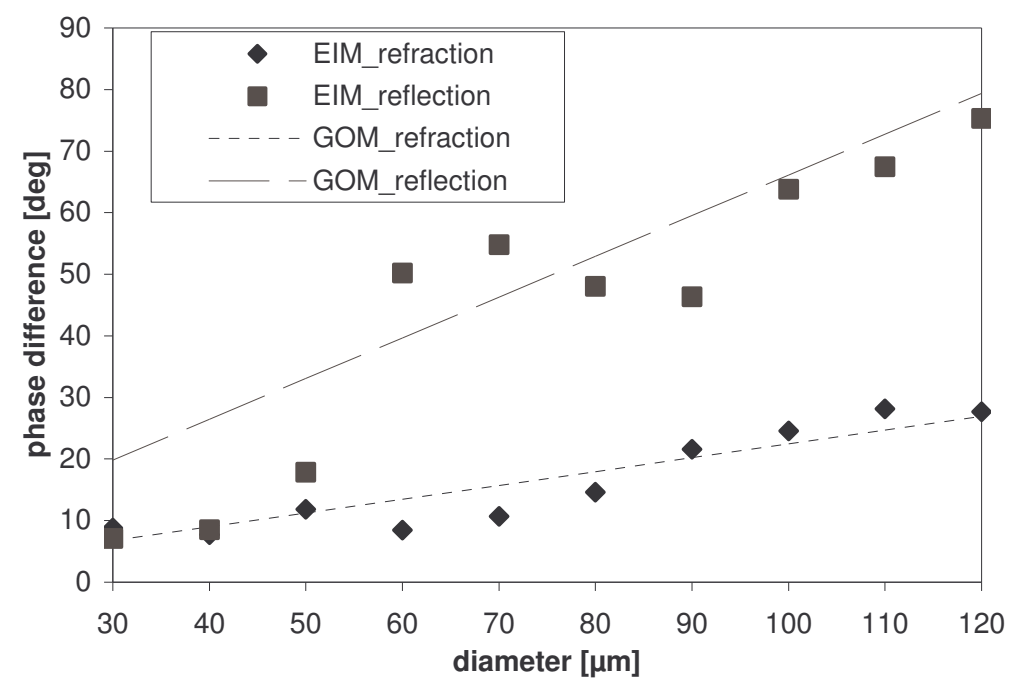

Fig. 14. Phase differences versus diameter of water droplet. 
1

2

3

4

5

6

7

8

10

11

12

13

14

15

16

17

18

19

20

21

22

23

24

25

26

27

28

29

30

31

32

33

34

35

36

37

38

39

40

41

42

43

44

45

46

47

48

49

50

51

52

53

54

55

56

57

58

59

60

61

62

63

64

65

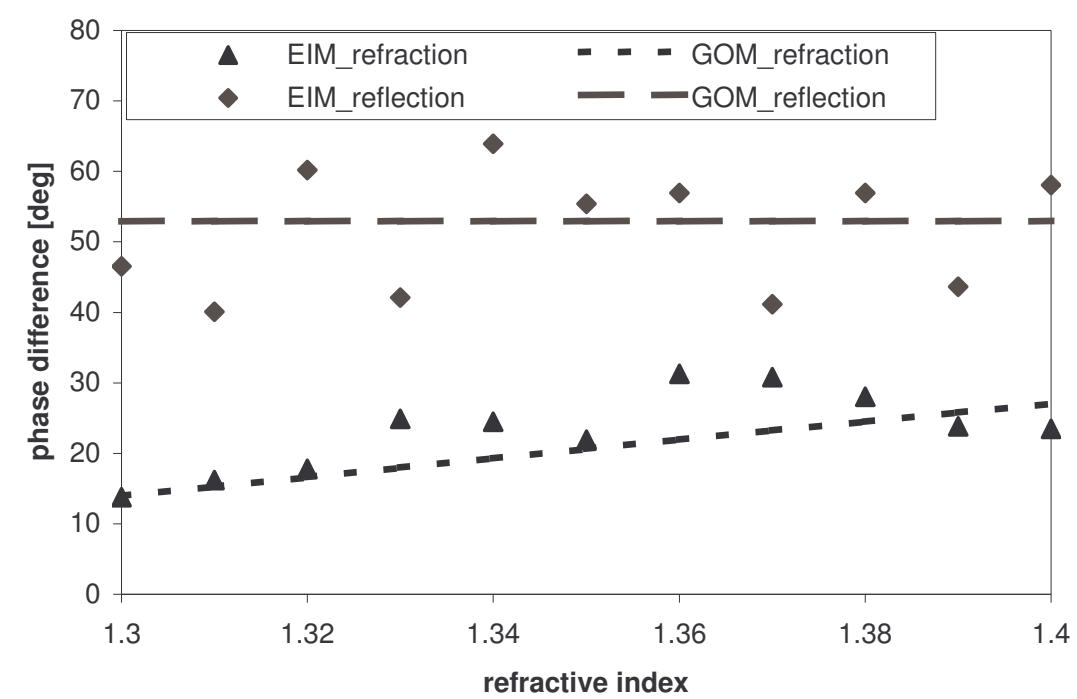

Fig. 15. Phase difference versus refractive index for a particle of diameter $80 \mu \mathrm{m}$. 
1

2

3

4

5

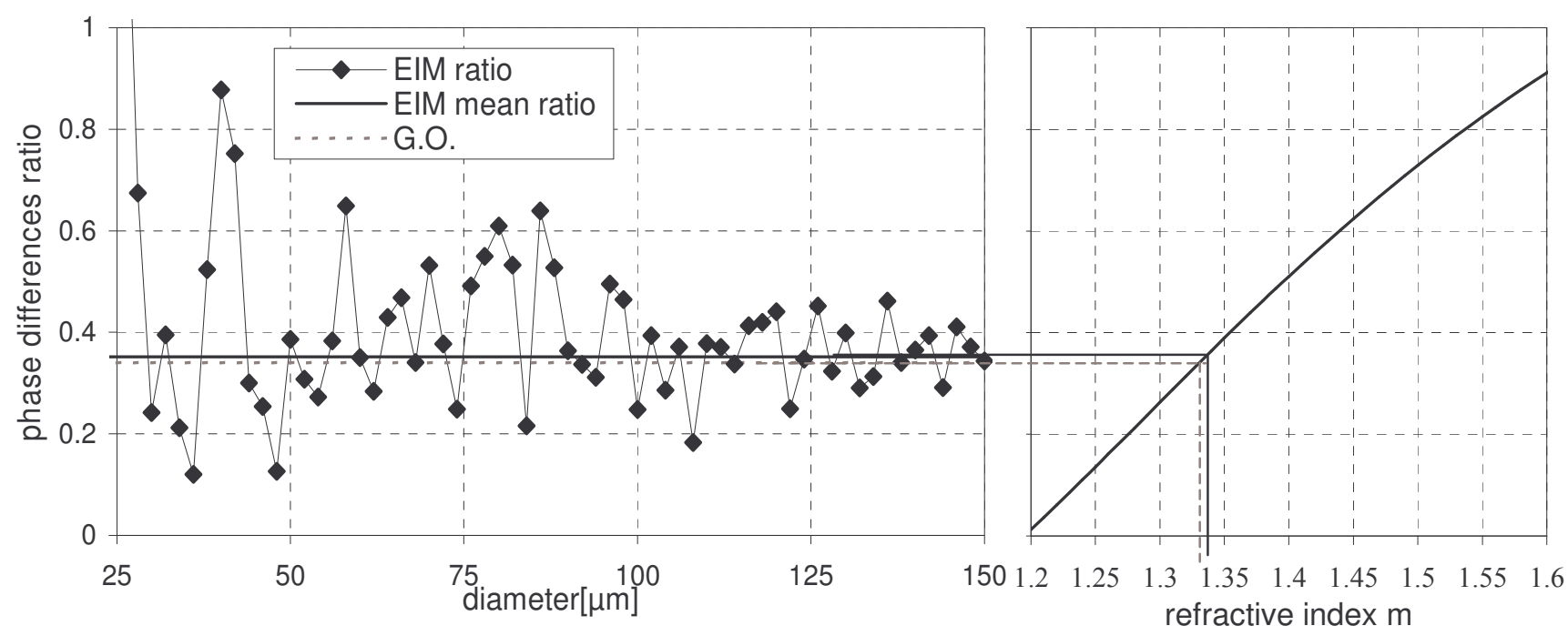

Fig. 16. Ratio of the two phase differences versus refractive index for a particle refractive index 1.33. 